1998-8

\title{
Nonlinear Optimal Control Design of a Missile Autopilot
}

Tim McLain

Brigham Young University - Provo, mclain@byu.edu

Randal W. Beard

Brigham Young University Department of Electrical Engineering

Follow this and additional works at: https://scholarsarchive.byu.edu/facpub

Part of the Mechanical Engineering Commons

\section{Original Publication Citation}

McLain, T. and Beard, R. Nonlinear Optimal Control Design of a Missile Autopilot, Proceedings of AIAA Guidance, Navigation, and Control Conference, AIAA-1998-4321, August 1998, Boston, Massachusetts.

\section{BYU ScholarsArchive Citation}

McLain, Tim and Beard, Randal W., "Nonlinear Optimal Control Design of a Missile Autopilot" (1998). Faculty Publications. 2106.

https://scholarsarchive.byu.edu/facpub/2106 


\section{NONLINEAR OPTIMAL CONTROL DESIGN OF A MISSILE AUTOPILOT}

\author{
Timothy W. McLain \\ Department of Mechanical Engineering \\ Brigham Young University \\ Provo, UT 84602 \\ tmclain@et . byu.edu
}

\author{
Randal W. Beard \\ Department of Electrical Engineering \\ Brigham Young University \\ Provo, UT 84602 \\ beard@ee.byu.edu
}

\begin{abstract}
The application of a new nonlinear optimal control strategy to the design of missile autopilots is presented. The control approach described and demonstrated here is based upon the numerical solution of the Hamilton-Jacobi-Bellman equation by Successive Galerkin Approximation. Using this approach, feedback controllers are computed by an iterative application of a numerical Galerkin-type PDE solver. Simulation results demonstrating the application of this approach to the design of a missile autopilot are presented.
\end{abstract}

\section{INTRODUCTION}

The dynamics of guided launch vehicles are inherently nonlinear due to inertial coupling, gravitational forces, aerodynamic effects, and actuator limits. Though autopilot designs are typically based on linearized dynamic models, modern missile systems often operate in flight regimes where nonlinearities significantly affect dynamic response.

Much of the recently published missile control literature focuses on the application of linear robust control methods to linearized missile models. ${ }^{1-3}$ System nonlinearities are dealt with by using gain scheduled controllers or by treating nonlinear terms as uncertain. While the use of modern linear design methods has yielded improvements in control system performance and robustness, the use of nonlinear control methods may provide a means to improve control precision and widen stability boundaries when flight must be conducted at high angles or angular rates where nonlinear effects are most extreme.

Such anticipated performance improvements has motivated research into nonlinear control design techniques. Among the methods that have been investigated are nonlinear optimal and robust control design approaches based upon the solutions to the Hamilton-Jacobi-Bellman (HJB) and HamiltonJacobi-Isaacs (HJI) equations respectively. The difficulty in using such approaches lies in approximating solutions to the HJB and HJI equations. Numerous approaches have been taken with mixed success. One approach that has been used for synthesis of optimal and robust autopilots requires the equations of motion of the nonlinear system to be approximated by a Taylor series expansion. ${ }^{4,5}$ Another approach that has been applied recently to robust missile autopilot design is to use the classical method of characteristics to approximate the HJI equation, which results in an open-loop control strategy. ${ }^{6}$

Using the synthesis method proposed here, feedback controllers are designed based on the full nonlinear dynamics of the system. There is no linearization involved, neither dynamic feedback linearization nor a priori linearization of the equations of motion. Optimal controllers are obtained by the numerical solution of the Hamilton-Jacobi-Bellman equation. The HJB equation is solved by successive application of a Galerkin-type solution strategy. The Successive Galerkin Approximation (SGA) strategy presented here was originated by Beard ${ }^{7}$ and has been applied to a variety of nonlinear systems. ${ }^{8,9}$

\section{CONTROL APPROACH}

For a system modeled by the nonlinear state equations

$$
\dot{x}=f(x)+g(x) u(x)
$$


a performance index $V(x)$ can be defined where

$$
V(x)=\int_{t}^{\infty}\left(l(x)+\|u(x)\|_{R}^{2}\right) d t
$$

$l(x)$ is a positive definite, zero-state observable function, and $R=R^{T}>0$. The control which minimizes the performance index is given by

$$
u^{*}(x)=-\frac{1}{2} R^{-1} g^{T}(x) \frac{\partial V^{*}}{\partial x}
$$

where $V^{*}(x)$ is a positive definite function that satisfies the Hamilton-Jacobi-Bellman equation:

$$
\frac{\partial V^{* T}}{\partial x} f+l-\frac{1}{4} \frac{\partial V^{* T}}{\partial x} g R^{-1} g^{T} \frac{\partial V^{*}}{\partial x}=0 .
$$

Unfortunately, obtaining a closed-form solution of Equation 4 for systems of the complexity of the missile system considered here is impossible. The approach for approximating the solution of the HJB equation presented here is to begin with a known control, $u^{(0)}$ (such as state feedback), that is stabilizing over a bounded domain of the state space $\Omega$ and to numerically compute successive approximations to the optimal control given by Equation 3 . These successive approximations are calculated in a two step process: (1) the nonlinear HJB partial differential equation is reduced to a sequence of linear PDEs known as the Generalized Hamilton Jacobi Bellman (GHJB) equation; and (2) the GHJB equation is solved through successive applications of Galerkin's method.

With the system operating under the initial feedback control law $u^{(0)}(x)$, the performance index of Equation 2 becomes

$$
V^{(0)}(x)=\int_{t}^{\infty}\left(l(x)+\left\|u^{(0)}(x)\right\|_{R}^{2}\right) d t .
$$

Taking the derivative of both sides of Equation 5 with respect to time and invoking the chain rule results in the partial differential equation

$$
\frac{\partial V^{(0) T}}{\partial x}\left[f+g u^{(0)}\right]+l+\left\|u^{(0)}\right\|_{R}^{2}=0 .
$$

Equations 5 and 6 show that $V^{(0)}$ is a Lyapunov function for the control $u^{(0)}$. Given $V^{(0)}$, the stability and robustness of $u^{(0)}$ can be improved by choosing a new control $u^{(1)}$ that makes

$\dot{V}^{(0)}=\frac{\partial V^{(0) T}}{\partial x}\left(f+g u^{(1)}\right)<\frac{\partial V^{(0) T}}{\partial x}\left(f+g u^{(0)}\right)<0$.
This is done by carrying out the following optimization:

$$
\begin{aligned}
u^{(1)} & =\arg { }_{u}^{\min }\left[\frac{\partial V^{(0) T}}{\partial x}(f+g u)+l+\|u\|_{R}^{2}\right] \\
& =-\frac{1}{2} R^{-1} g^{T} \frac{\partial V^{(0)}}{\partial x} .
\end{aligned}
$$

Equations 6 and 7 can be generalized to form the basis for an iterative solution process where

$$
\begin{gathered}
\frac{\partial V^{(i) T}}{\partial x}\left[f+g u^{(i)}\right]+l+\left\|u^{(i)}\right\|_{R}^{2}=0 \\
u^{(i+1)}=-\frac{1}{2} R^{-1} g^{T} \frac{\partial V^{(i)}}{\partial x} .
\end{gathered}
$$

The problem of determining the optimal control is simplified to solving the GHJB equation, Equation 8, in conjunction with the optimal control law of Equation 9. Because the GHJB is linear its numerical solution is straightforward relative to the HJB equation.

The solution of Equations 8 and 9 is carried out by first starting with a known stabilizing control, $u^{(0)}$, and then solving the GHJB equation using a computational Galerkin method to find $V^{(0)} \cdot V^{(0)}$ is then used to calculate $u^{(1)}$ and so on. Using this approach, successive values for $V^{(i)}$ and $u^{(i)}$ are computed, which for sufficiently large $i$, have been shown to converge monotonically to the optimal values $V^{*}$ and $u^{*} \cdot{ }^{11}$

Using the Galerkin method, ${ }^{10}$ it is first assumed that the cost function $V^{(i)}$ can be approximated using an infinite series of known basis functions $\phi_{j}(x)$ that are continuous and defined everywhere on the domain of the state space of interest $(\Omega)$ :

$$
V^{(i)}=\sum_{j=1}^{\infty} c_{j}^{(i)} \phi_{j}^{(i)}(x) .
$$

From an implementation standpoint, using an infinite number of terms in the approximation is not practical. Therefore, the approximation for $V^{(i)}$ is carried one step further by considering only the first $N$ terms of the infinite series:

$$
V_{N}^{(i)}=\sum_{j=1}^{N} c_{j}^{(i)} \phi_{j}^{(i)}(x) .
$$

The accuracy of this approximation to the assumed solution $V^{(i)}$ depends both on the number and characteristics of the basis functions chosen to form the approximation. 
Examining Equation 8, it can be seen that for $V^{(i)}$ satisfying the GHJB equation, the left-hand side evaluates to zero. Similarly, when the approximation to $V_{N}^{(i)}$ given by Equation 10 is substituted into Equation 8, a non-zero left-hand side is obtained. The error $e^{(i)}$ resulting from this approximation can be expressed as

$$
e^{(i)}(x)=\left(\sum_{j=1}^{N} c_{j}^{(i)} \frac{\partial \phi_{j}^{T}}{\partial x}\right)\left[f+g u^{(i)}\right]+l+\left\|u^{(i)}\right\|_{R}^{2} .
$$

Using the Galerkin approach, values for the basis function coefficients $c_{j}^{(i)}$ are determined such that the error $e^{(i)}$ is minimized. This is done by projecting the error onto the $N$ basis functions $\left(\phi_{1}, \phi_{2}, \ldots, \phi_{N}\right)$ by taking the inner product of the error and the basis on $\Omega$ and setting it to zero.* The result is $N$ linear equations in the $N$ unknown coefficients $c_{1}^{(i)}, c_{2}^{(i)}, \ldots, c_{N}^{(i)}$ which are expressed compactly as

$$
\left\langle e^{(i)}(x), \phi_{j}(x)\right\rangle_{\Omega}=0, \quad j=1 \ldots N .
$$

Equation 12 can be expressed in more detail in matrix form as

$$
c^{(i)}=\left(A_{1}+A_{2}^{(i)}\right)^{-1}\left(b_{1}+b_{2}^{(i)}\right)
$$

where

$$
\begin{gathered}
A_{1}=\left[\begin{array}{ccc}
\left\langle\frac{\partial \phi_{1}^{T}}{\partial x} f, \phi_{1}\right\rangle_{\Omega} & \cdots & \left\langle\frac{\partial \phi_{N}^{T}}{\partial x} f, \phi_{1}\right\rangle_{\Omega} \\
\vdots & \ddots & \vdots \\
\left\langle\frac{\partial \phi_{1}^{T}}{\partial x} f, \phi_{N}\right\rangle_{\Omega} & \cdots & \left\langle\frac{\partial \phi_{N}^{T}}{\partial x} f, \phi_{N}\right\rangle_{\Omega}
\end{array}\right] \\
A_{2}^{(i)}=\left[\begin{array}{ccc}
\left\langle\frac{\partial \phi_{1}^{T}}{\partial x} g u^{(i)}, \phi_{1}\right\rangle_{\Omega} & \cdots & \left\langle\frac{\partial \phi_{N}^{T}}{\partial x} g u^{(i)}, \phi_{1}\right\rangle_{\Omega} \\
\vdots & \ddots & \vdots \\
\left\langle\frac{\partial \phi_{1}^{T}}{\partial x} g u^{(i)}, \phi_{N}\right\rangle_{\Omega} & \cdots & \left\langle\frac{\partial \phi_{N}^{T}}{\partial x} g u^{(i)}, \phi_{N}\right\rangle_{\Omega}
\end{array}\right] \\
b_{1}=-\left[\begin{array}{c}
\left\langle l, \phi_{1}\right\rangle_{\Omega} \\
\vdots \\
\left\langle l, \phi_{N}\right\rangle_{\Omega}
\end{array}\right]
\end{gathered}
$$

*The inner product of two functions $f(x)$ and $g(x)$ is defined as

$$
\langle f(x), g(x)\rangle_{\Omega} \triangleq \int_{\Omega} f(x) g(x) d x
$$

$$
b_{2}^{(i)}=-\left[\begin{array}{c}
\left\langle\left\|u^{(i)}\right\|_{R}^{2}, \phi_{1}\right\rangle_{\Omega} \\
\vdots \\
\left\langle\left\|u^{(i)}\right\|_{R}^{2}, \phi_{N}\right\rangle_{\Omega}
\end{array}\right] .
$$

With the coefficients $c^{(i)}=\left[\begin{array}{llll}c_{1}^{(i)} & c_{2}^{(i)} & \cdots & c_{N}^{(i)}\end{array}\right]^{T}$ calculated from Equation 13, the control associated with the $(i+1)^{t h}$ iteration can be determined:

$$
u_{N}^{(i+1)}=-\frac{1}{2} R^{-1} g^{T}\left(\sum_{j=1}^{N} c_{j}^{(i)} \frac{\partial \phi_{j}}{\partial x}\right) .
$$

Expressions for $A_{2}^{(i)}$ and $b_{2}^{(i)}$ above indicate that their integrals require recalculation at each iteration step due to their dependence on $u^{(i)}$. This is not necessarily the case. By taking advantage of Equation $14, A_{2}^{(i)}$ and $b_{2}^{(i)}$ can be evaluated in an iterative fashion as well. $A_{2}^{(0)}$ and $b_{2}^{(0)}$ are calculated using the expressions above, while values for subsequent iterations can be calculated as follows:

$$
\begin{gathered}
A_{2}^{(i)}=-\frac{1}{2} \sum_{j=1}^{N} c_{j}^{(i-1)} M_{j} \\
b_{2}^{(i)}=-\frac{1}{4}\left[\begin{array}{c}
c^{(i-1) T} M_{1} c^{(i-1)} \\
c^{(i-1) T} M_{2} c^{(i-1)} \\
\vdots \\
c^{(i-1) T} M_{N} c^{(i-1)}
\end{array}\right]
\end{gathered}
$$

where

$$
\begin{gathered}
\left\{M_{i}\right\}_{1}^{N}=\left[\begin{array}{ccc}
m_{i}(1,1) & \ldots & m_{i}(1, N) \\
\vdots & \ddots & \vdots \\
m_{i}(N, 1) & \ldots & m_{i}(N, N)
\end{array}\right] \\
m_{i}(j, k)=\left\langle\frac{\partial \phi_{k}^{T}}{\partial x} g R^{-1} g^{T} \frac{\partial \phi_{i}}{\partial x}, \phi_{j}\right\rangle_{\Omega} .
\end{gathered}
$$

From a computational point of view, evaluating the $n$-dimensional integrals required to compute the basis function coefficients is quite demanding. Previous work has shown that this computational burden can be eased significantly through the use of basis functions that are separable. ${ }^{12}$ It is important to note these coefficient calculations are carried out prior to implementation. The calculations carried out during operation are straightforward and easily done in real time. 


\section{MISSILE SYSTEM MODEL}

This paper addresses the design of an autopilot for the pitch-plane motion of a tail-controlled missile system. The mathematical model for the system under consideration is based upon the model originally presented by Reichert ${ }^{2,13}$ for a missile traveling at Mach 3 at an altitude of 20,000 ft. Figure 1 shows a schematic representation of the missile and the coordinates used to represent its motion.

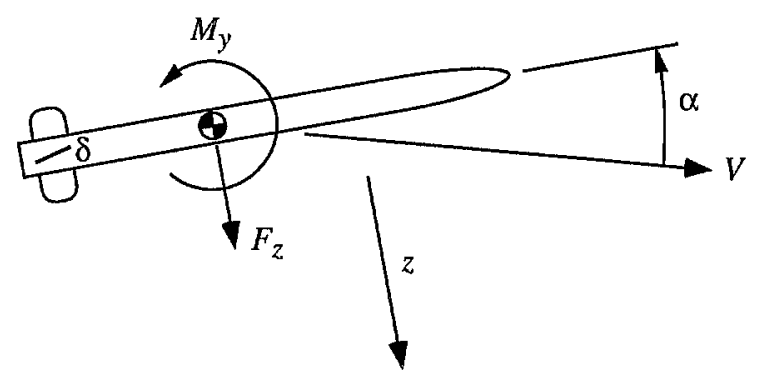

Figure 1: Missile System Pitch-Plane Schematic

The equations of motion which describe the dynamics of the missile are given by

$$
\begin{aligned}
\dot{q} & =\frac{M_{y}}{I_{y}} \\
\dot{\alpha} & =\frac{\cos ^{2}(\alpha)}{m U} F_{z}+q \\
\ddot{\delta} & =-2 \zeta \omega_{n} \dot{\delta}-\omega_{n}^{2} \delta+\omega_{n}^{2} \delta_{c}
\end{aligned}
$$

where

$$
\begin{array}{ll}
q & =\text { pitch rate, deg } / \mathrm{s} \\
\alpha & =\text { angle of attack, deg } \\
\delta & =\text { tail fin deflection, deg } \\
\delta_{c} & =\text { commanded tail fin deflection, } \mathrm{deg} \\
M_{y} & =C_{m}(\alpha, \delta) Q S d, \mathrm{ft}-\mathrm{lb} \\
F_{z} & =C_{n}(\alpha, \delta) Q S, \mathrm{lb} \\
I_{y} & =\text { pitch moment of inertia, } 182.5 \mathrm{slug}-\mathrm{ft}^{2} \\
m & =\text { mass of missile, } 13.98 \mathrm{slug} \\
U & =\text { longitudinal velocity, } V \cos (\alpha), \mathrm{ft} / \mathrm{s} \\
\zeta & =\text { fin actuator damping ratio, } 1.4 \\
\omega_{n} & =\text { fin actuator natural frequency, } 5 \mathrm{~Hz} \\
M & =\text { Mach number, } 3 \\
Q & =\text { dynamic pressure, } 0.7 \rho M^{2}, \mathrm{lb} / \mathrm{ft}^{2} \\
S & =\text { reference area, } 0.44 \mathrm{ft}^{2} \\
d & =\text { diameter, } 0.75 \mathrm{ft} \\
\rho & =\text { static pressure at } 20,000 \mathrm{ft}, 973.3 \mathrm{lb} / \mathrm{ft}^{2} \\
s_{s} & =\text { speed of sound at } 20,000 \mathrm{ft}, 1036.4 \mathrm{ft} / \mathrm{s} \\
V & =\text { velocity of missile, } M s_{s}, \mathrm{ft} / \mathrm{s} .
\end{array}
$$

The aerodynamic coefficients, $C_{n}$ and $C_{m}$ can be represented by the following polynomial functions of $\alpha$ and $\delta$ :

$$
\begin{aligned}
C_{n} & =a_{1} \alpha^{3}+a_{2} \alpha|\alpha|+a_{3} \alpha+a_{4} \delta \\
a_{1} & =.000103 \\
a_{2} & =-.00945 \\
a_{3} & =-.170 \\
a_{4} & =-.034 \\
C_{m} & =b_{1} \alpha^{3}+b_{2} \alpha|\alpha|+b_{3} \alpha+b_{4} \delta \\
b_{1} & =.000215 \\
b_{2} & =-.0195 \\
b_{3} & =.051 \\
b_{4} & =-.206 .
\end{aligned}
$$

For this system, the output of interest is the acceleration of the center of mass in the direction normal to the longitudinal axis of the missile (in units of $g$ ), $\eta_{z}$ :

$$
\eta_{z}=\frac{F_{z}}{m g} .
$$

The control synthesis algorithm developed above is for a regulator. In other words, all of the states and the control must go to zero as time goes to infinity. The missile autopilot design objective is to control the $z$-axis acceleration to a non-zero reference value. To do so, several of the states (namely $q, \alpha$, and $\delta$ ) and the control $\left(\delta_{c}\right)$ must be non-zero in steady state. For the SGA algorithm to be applicable, a change of variables must take place.

With the missile operating at the desired steadystate acceleration $\eta_{z_{d}}$, the values of the states, $\left[q_{s s} \alpha_{s s} \dot{\delta}_{s s} \delta_{s s}\right]^{T}$, and the control, $\delta_{c_{s s}}$, required to cause this acceleration can be found by numerically solving the nonlinear system of equations resulting from the state equations and output equation when the state derivatives are set to zero

$$
\begin{aligned}
0 & =\frac{M_{y_{s s}}}{I_{y}} \\
0 & =\frac{\cos ^{2}\left(\alpha_{s s}\right)}{m U} F_{z_{s s}}+q_{s s} \\
0 & =-\omega_{n}^{2} \delta_{s s}+\omega_{n}^{2} \text { delt } a_{c_{s s}} \\
\eta_{z_{d}} & =\frac{F_{z_{s s}}}{m} .
\end{aligned}
$$

with $M_{y_{s s}}$ and $F_{z_{s s}}$ determined from the relations for $M_{y}$ and $F_{z}$ defined above.

By performing the change of variables

$$
x=\left[\begin{array}{l}
x_{1} \\
x_{2} \\
x_{3} \\
x_{4}
\end{array}\right]=\left[\begin{array}{c}
q-q_{s s} \\
\alpha-\alpha_{s s} \\
\dot{\delta}-\dot{\delta}_{s s} \\
\delta-\delta_{s s}
\end{array}\right]
$$


for the states and $u=\delta_{c}-\delta_{c_{s s}}$ for the control on the equations of motion, a new set of state equations results where a stabilizing control will cause $\eta_{z} \rightarrow$ $\eta_{z_{d}}, x \rightarrow 0$, and $u \rightarrow 0$ as $t \rightarrow \infty$, thereby enabling the application of the SGA algorithm.

\section{CONTROL SYNTHESIS AND IMPLEMENTATION}

With the model described above, the optimal feedback control can be synthesized using the SGA algorithm. With $f(x)$ and $g(x)$ coming directly from the equations of motion and $l(x)$ and $R$ being determined by the control designer, the domain of the states $\Omega$, the basis functions $\left\{\phi_{j}(x)\right\}_{1}^{N}$, and the initial control law $u^{(0)}$ remain to be determined.

The domain of possible values for the states is determined both by the physical capabilities of the system and the likely deviation of the states from their nominal value of zero. For the results presented here, $\Omega$ was defined to be

$$
\begin{aligned}
-200 \mathrm{deg} / \mathrm{s} & \leq x_{1} \leq 200 \mathrm{deg} / \mathrm{s} \\
-25 \mathrm{deg} & \leq x_{2} \leq 25 \mathrm{deg} \\
-800 \mathrm{deg} / \mathrm{s} & \leq x_{3} \leq 800 \mathrm{deg} / \mathrm{s} \\
-25 \mathrm{deg} & \leq x_{4} \leq 25 \mathrm{deg} .
\end{aligned}
$$

Proper selection of the basis functions $\left\{\phi_{j}(x)\right\}_{1}^{N}$ is critical to the design of the optimal controller. The basis functions must be able to capture the nonlinear dynamics behavior of the system in order to approximate the cost function $V^{(i)}$ with sufficient accuracy. By doing so, a control law can be synthesized that will compensate for the nonlinear dynamics of the system.

Two practical issues affect the selection of basis functions. First, the terms that show up in the control are determined by $g$ and $\frac{\partial \phi_{j}}{\partial x}$ (see Equation 14). To regulate the states of a system, some care must be taken in the selection of basis functions to ensure that their partials with respect to the states produce functions of the states that will ultimately show up in the control law. This is especially true when $g$ has a significant number of zero terms. Second, basis functions must be chosen so that the matrix $\left(A_{1}+A_{2}^{(i)}\right)$ of Equation 13 is invertible. When basis functions are chosen arbitrarily without considering the dynamics of the system, the rank of the coefficient matrix to be inverted can become an issue. For this problem, the following basis functions were used:

$$
\begin{array}{r}
\left\{\phi_{j}\right\}_{j=1}^{12}=\left\{x_{1}^{2}, x_{1} x_{2}, x_{2}^{2}, x_{1} x_{3}, x_{2} x_{3}, x_{3}^{2}, x_{1} x_{4},\right. \\
\left.x_{2} x_{4}, x_{3} x_{4}, x_{4}^{2}, x_{2}\left|x_{2}\right| x_{3}, x_{2}^{3} x_{3}\right\} .
\end{array}
$$

The initial stabilizing control was designed by linearizing the equations of motion about zero angle of attack and using pole placement. For this problem, the following initial control was used:

$$
\begin{aligned}
u^{(0)} & =.08 q+.38 \alpha+.37 \eta_{z_{d}} \\
& =.08\left(x_{1}+q_{s s}\right)+.38\left(x_{2}+\alpha_{s s}\right)+.37 \eta_{z_{d}} .
\end{aligned}
$$

\section{RESULTS}

Using the SGA algorithm, four different optimal controllers were designed and implemented: Two different cost functions, $l(x)$, were tested for two different acceleration commands. Since the system equations of motion are affected by the choice of the acceleration reference command (because of the change of variables), separate control laws must be synthesized for different acceleration commands. For each of the controllers designed, the weight on the control was set to one $(R=1)$.

Table 1 shows the weighting functions and resulting SGA control laws for each of the four controllers, as well as the value of the cost function for the initial state feedback control $V^{(0)}$ and the minimized cost function value for each of the SGA controller designs. As expected, the cost function value is significantly reduced for the SGA optimal designs.

The first cost function on the states was chosen simply to be the square of the error between the desired and actual accelerations:

$$
l_{1}(x)=\left(\eta_{z_{d}}-\eta_{z}\right)^{2}
$$

where $\eta_{z}$ is calculated from

$$
\begin{aligned}
\eta_{z}= & \frac{Q S}{m g}\left[a_{1}\left(x_{2}+\alpha_{s s}\right)^{3}+a_{2}\left(x_{2}+\alpha_{s s}\right)\left|x_{2}+\alpha_{s s}\right|\right. \\
& \left.+a_{3}\left(x_{2}+\alpha_{s s}\right)+a_{4}\left(x_{4}+\delta_{s s}\right)\right] .
\end{aligned}
$$

The use of this cost function demonstrates that the algorithm is not limited to the use of cost functions that are quadratic in the state variables. Using this cost function two different acceleration step commands were tested: $-10 g$ and $25 g$. Figure 2 shows the response of the missile to the $-10 \mathrm{~g}$ command, while Figure 3 shows the response of the missile to the $25 \mathrm{~g}$ command.

For both the $-10 g$ and the $25 g$ commands, the actual accelerations settle to the desired values as 
Table 1: SGA Optimal Control Designs

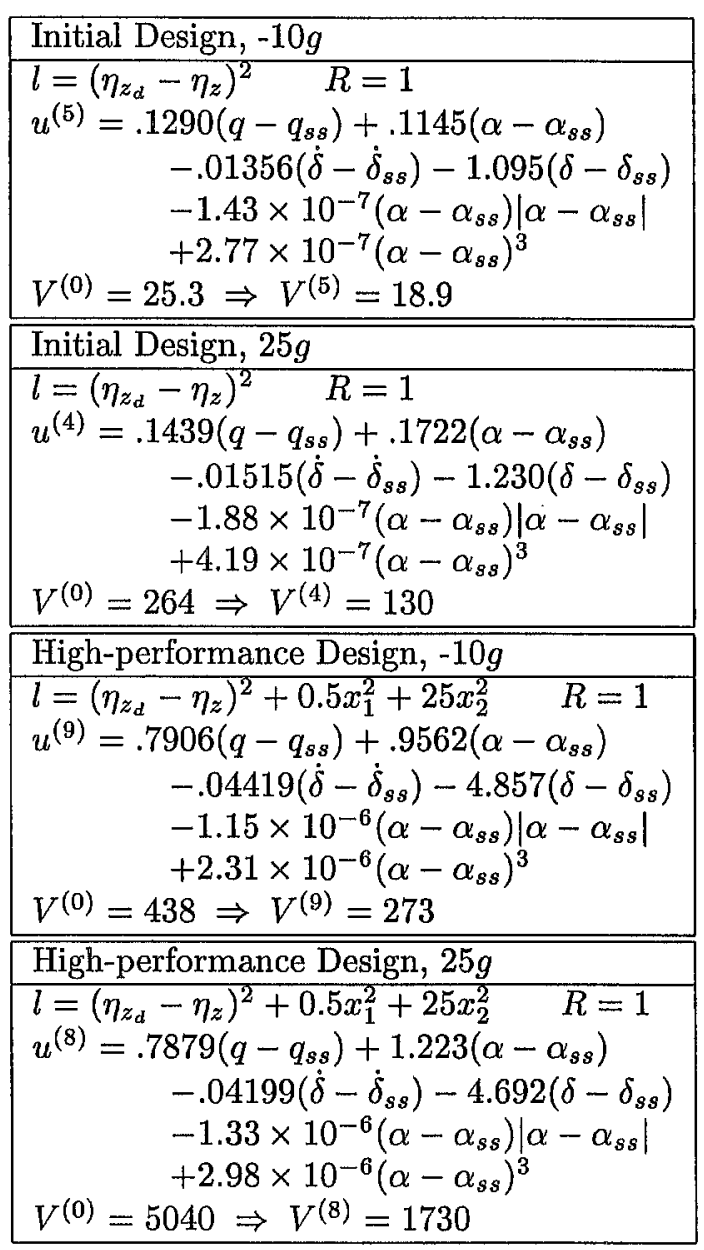
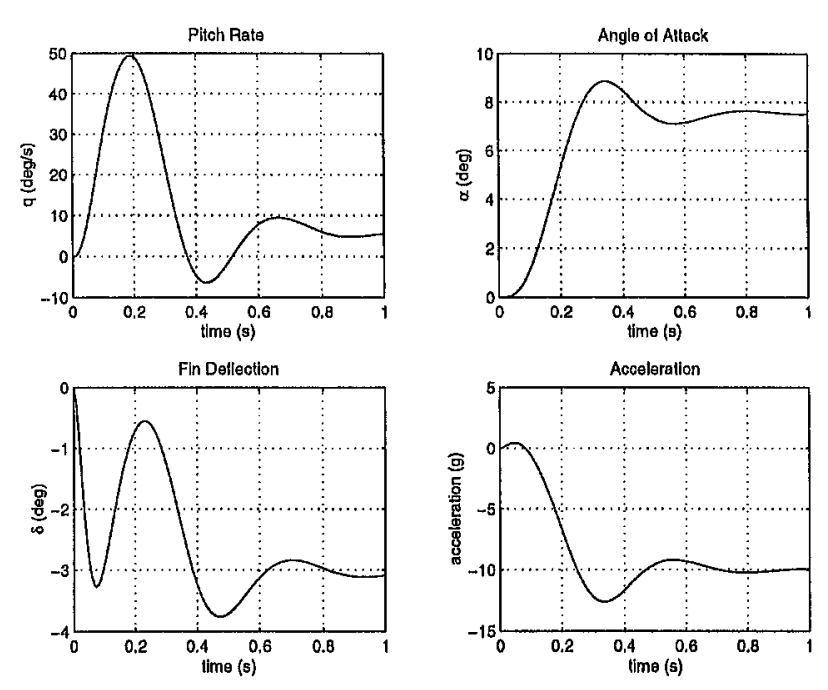

Figure 2: Initial Missile Response: $-10 g$ Command
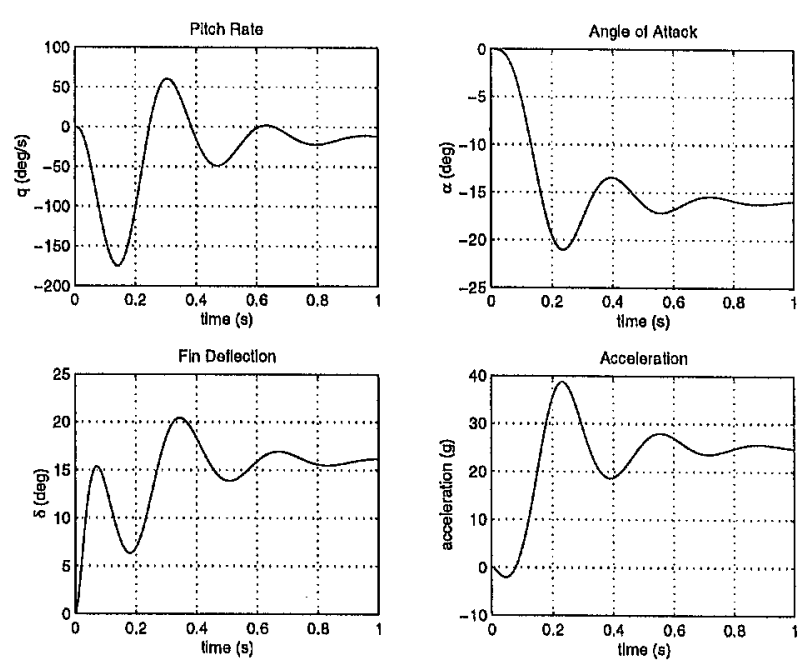

Figure 3: Initial Missile Response: $25 g$ Command

expected. However, in both cases the overshoot and settling time are larger than desired (especially the $25 \mathrm{~g}$ case). This was remedied by changing the cost function to have additional weights on pitch-rate and angle-of-attack errors:

$$
l_{2}(x)=\left(\eta_{z_{d}}-\eta_{z}\right)^{2}+0.5 x_{1}^{2}+25 x_{2}^{2} .
$$

The weight on pitch-rate error increases the damping in the system, while the weight on angle-of-attack error helps improve the speed of response. This is demonstrated in Figures 4 and 5. In both cases, the overshoot and settling times are reduced significantly resulting in very good response to the acceleration step commands. As expected, the peak control efforts required (as reflected by the fin deflection angle histories) are increased over the initial designs.

As can be seen in Table 1, the resulting control laws place the highest gains on the linear feedback terms. Also of interest is the relative insensitivity of the control law designs to changes in the magnitude of the acceleration command. While the the control law gains for the $-10 \mathrm{~g}$ and $25 \mathrm{~g}$ designs are different for both initial and final designs, their difference is not as large as one might anticipate. The most significant variations are in the gain on angle of attack. As expected, when the $-10 g$ initial and $-10 g$ final designs are compared, the gains are significantly higher for the high-performance design. The same is true when the $25 g$ initial and $25 g$ final designs are compared.

The results demonstrate the ability to develop and tune optimal controllers for nonlinear systems. Through the proper selection of basis functions and appropriate tuning of $l(x)$ and $R$, the behavior of 

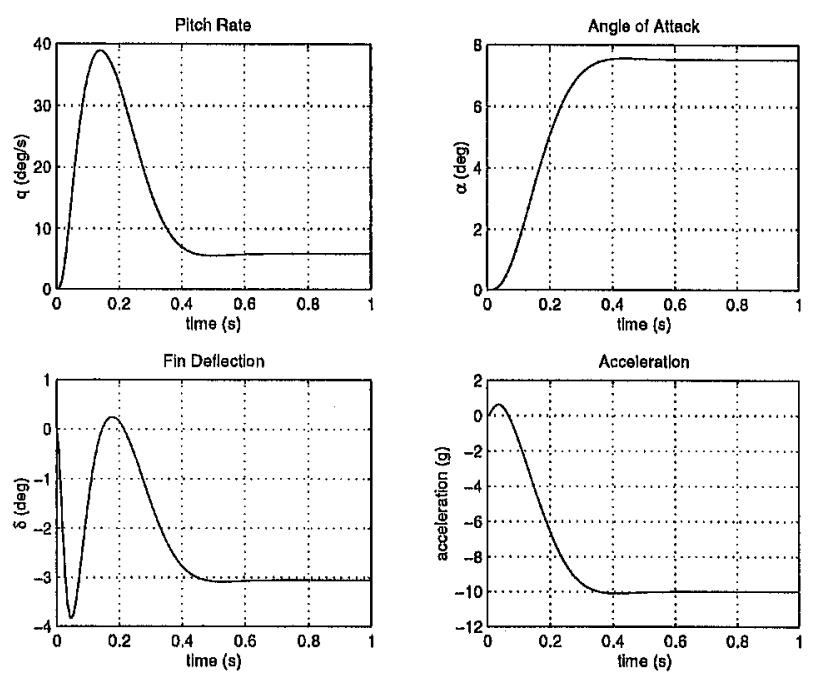

Figure 4: Improved Missile Response: $-10 g$ Command
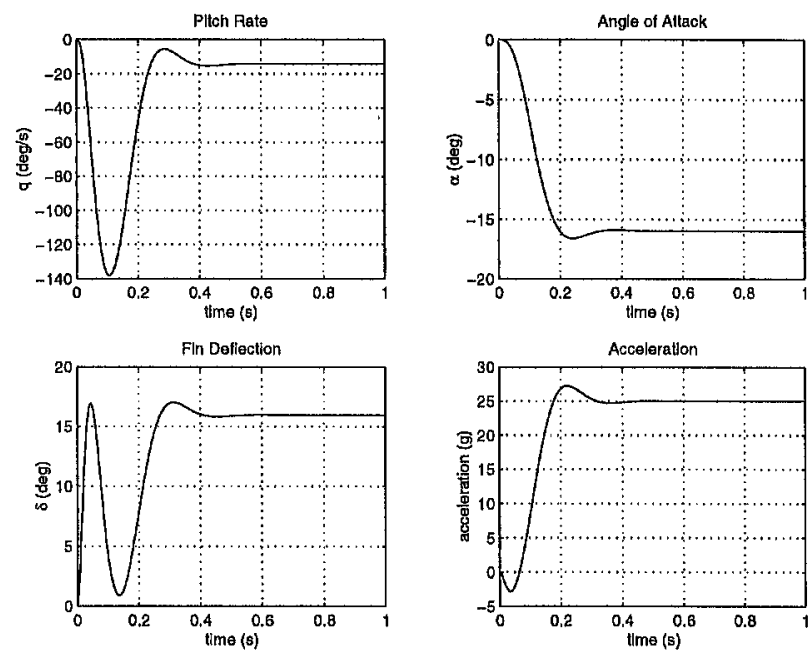

Figure 5: Improved Missile Response: $25 \mathrm{~g}$ Command

the controlled system can be altered to meet the demands of a specific application. This can be done by considering the full nonlinear dynamics of the system without the need for linearization of the equations of motion.

\section{CONCLUSIONS}

The Successive Galerkin Approximation algorithm for numerically solving the Hamilton-JacobiBellman equation has been presented. Using this synthesis algorithm, nonlinear optimal controllers have been designed for the pitch-axis autopilot of an agile missile. These feedback controllers were de- signed taking into account the full nonlinear dynamics of the missile system. Simulation results demonstrated the ability to tune the performance of the system by adjusting weighting functions and to track acceleration commands of different magnitudes.

\section{REFERENCES}

[1] G. Gu, J. R. Cloutier, and G. Kim, "Gain scheduled missile autopilot design using observer-based $H_{\infty}$ control", in Proceedings of the American Control Conference, 1995, pp. 1951-1955.

[2] G. J. Balas, R. Lind, and A. Packard, "Optimally scaled $H_{\infty}$ full information control synthesis with real uncertainty", AIAA Journal of Guidance, Control, and Dynamics, vol. 19, no. 4, pp. 854-862, 1996.

[3] S.M. Yang and N.H. Huang, "Application of $H_{\infty}$ control to pitch autopilot of missiles", IEEE Transactions on Aerospace and Electronic Systems, vol. 32, no. 1, pp. 426-433, 1996.

[4] W. L. Garrard, D. F. Enns, and S. A. Snell, "Nonlinear feedback control of highly manoeuvrable aircraft", International Journal of Control, vol. 56, no. 4, pp. 799-812, 1992.

[5] J. Huang and C.-F. Lin, "Numerical approach to computing nonlinear $H_{\infty}$ control laws", AIAA Journal of Guidance, Control, and Dynamics, vol. 18, no. 5, pp. 989-996, 1995.

[6] K. A. Wise and J. L. Sedwick, "Nonlinear $H_{\infty}$ optimal control for agile missiles", AIAA Journal of Guidance, Control, and Dynamics, vol. 19, no. 1, pp. 157-165, 1996.

[7] R. W. Beard, Improving the Closed-Loop Performance of Nonlinear Systems, $\mathrm{PhD}$ thesis, Rensselaer Polytechnic Institute, Troy, NY $12180,1995$.

[8] R. Beard, G. Saridis, and J. Wen, "Improving the performance of stabilizing controls for nonlinear systems", IEEE Control Systems Magazine, vol. 16, no. 5, pp. 27-35, 1996.

[9] T. W. McLain and R. W. Beard, "Nonlinear optimal control of a hydraulically actuated positioning system", in Proceedings of the ASME International Mechanical Engineering Congress and Exposition, Nov 1997. 
[10] C.A.J. Fletcher, Computational Galerkin Methods, Springer-Verlag, 1984.

[11] G.N. Saridis and J. Balaram, "Suboptimal control for nonlinear systems", Control Theory and Advanced Technology, vol. 2, pp. 547-562, Sep 1986.

[12] J. Lawton and R. Beard, "Numerically efficient approximations to the Hamilton-JacobiBellman equation", in Proceedings of the American Control Conference, Philadelphia, PA, 1998.

[13] R. T. Reichert, "Robust autopilot design using $\mu$-synthesis", in Proceedings of the American Control Conference, 1990, pp. 2368-2373. 